\title{
Inclusive Higgs production via double pomeron exchange
}

\section{Christophe Royon*}

DAPNIA/SPP, Commissariat à l'Energie Atomique, Saclay,

F-91191 Gif-sur-Yvette Cedex

Brookhaven National Laboratory, Upton, New York, 11973;

University of Texas, Arlington, Texas, 76019

E-mail: royon@hep. saclay.cea.fri

Abstract: We evaluate inclusive Higgs boson and dijet cross-sections at the Tevatron collider via double pomeron exchange. Such inclusive processes, normalized to the observed dijet rate observed at run I by the CDF collaboration, noticeably increase the predictions for Higgs production in diffractive events at the Tevatron Run II.

\section{Theoretical framework}

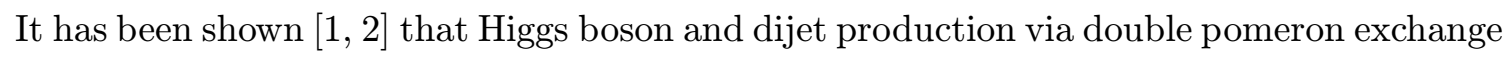
may be non negligeable. Our aim is to give predictions based on inclusive Higgs boson and dijet production at the Tevatron collider via double pomeron exchange [iji]. So far, the predictions have been based on exclusive production without accompanying radiation, which is indeed present, e.g. in double pomeron dijet production at the Tevatron [i

In the following we use as a starting point the Bialas-Landshoff exclusive model for Higgs boson and heavy flavor jet production [i]. We modify it in order to take into account inclusive Higgs boson and dijet production [3i3]

Let us introduce the formulae for inclusive Higgs boson and dijet production crosssections via double pomeron exchange.

$$
\begin{aligned}
d \sigma_{H}^{i n c l} & =C_{H}\left(\frac{x_{1}^{g} x_{2}^{g} s}{M_{H}^{2}}\right)^{2 \epsilon} \delta\left(\xi_{1} \xi_{2}-\frac{M_{H}^{2}}{x_{1}^{g} x_{2}^{g} s}\right) \prod_{i=1,2}\left\{G_{P}\left(x_{i}^{g}, \mu\right) \frac{d x_{i}^{g}}{x_{i}^{g}} \frac{d \xi_{i}}{\xi_{i}} d^{2} v_{i} \xi_{i}^{\alpha^{\prime} v_{i}^{2}} \exp \left(-2 \lambda v_{i}^{2}\right)\right\}, \\
d \sigma_{J J}^{i n c l} & \sim\left(\frac{x_{1}^{g} x_{2}^{g} s}{M_{\bar{J} J}^{2}}\right)^{2 \epsilon} F_{J J} \prod_{i=1,2}\left\{G_{P}\left(x_{i}^{g}, \mu\right) \frac{d x_{i}^{g}}{x_{i}^{g}} \frac{d \xi_{i}}{\xi_{i}} d^{2} v_{i} d^{2} k_{i} \xi_{i}^{\alpha^{\prime} v_{i}^{2}} \exp \left(-2 \lambda v_{i}^{2}\right)\right\}
\end{aligned}
$$

\footnotetext{
${ }^{*}$ Speaker.
} 
where $x_{1}^{g}, x_{2}^{g}$ define the fraction of the Pomerons' momentum carried by the gluons involved in the hard process, see Fig.1, and $G_{P}\left(x_{1,2}^{g}, \mu\right)$, are, up to a normalization, the gluon structure function in the Pomerons extracted from HERA experiments, see [5. $\mu^{2}$ is the hard scale (for simplicity kept fixed at $75 \mathrm{GeV}^{2}$, the highest value studied at HERA; we neglect the small [5] contribution of quark initiated processes in the Pomeron). By construction of our model, the known formulae for exclusive channels in Ref.[i] $g g \rightarrow g g$, not included in $\left.\left[\begin{array}{l}{[\bar{i}} \\ {[}\end{array}\right]\right)$ are recovered by the substitution $G_{P}\left(x_{i}^{g}, \mu\right) \rightarrow \delta\left(\bar{x}_{i}^{g}-1\right)$.

The formulae (1) $\left.\overline{1}_{1}^{1} \overline{1}_{1}\right)$ are written for a Higgs boson of mass $M_{H}$ and two jets (of total mass $\left.M_{\bar{J} J}\right)$, respectively. The Pomeron trajectory is $\alpha(t)=1+\epsilon+\alpha^{\prime} t\left(\epsilon \sim .08, \alpha^{\prime} \sim .25 \mathrm{GeV}^{-2}\right)$, $\xi_{1,2}(<.1)$ are the Pomerons' fraction of longitudinal momentum, $v_{1,2}$, the 2-transverse momenta of the outgoing $p, \bar{p}, k_{1,2}$, those of the outgoing quark jets, $\lambda \sim 4 \mathrm{GeV}^{-2}$ the slope of the Pomeron $p \bar{p}$ coupling. [6]'].

The dijet cross-section $\sigma_{J J}$ depends on the $g g \rightarrow \bar{Q}^{f} Q^{f}$ and $g g \rightarrow g g$ cross-sections

The physical origin of ourformulae ( $(\overline{1} \cdot \overline{1})$ extended to the inclusive case is the following: Since the overall partonic configuration is produced initially by the long-range, soft double pomeron interaction, we assume that, up to a normalization, the inclusive cross-section is the convolution of the "hard" partons $\rightarrow$ Higgs boson, partons $\rightarrow$ jets subprocesses by the Pomeron structure function into gluons, see Fig.1. The expected factorization breaking of hadroproduction will appear in the normalization through a renormalization of the Pomeron fluxes, which are not the same as in hard diffraction at HERA. Indeed, this ansatz remarkably reproduces the dijet mass fraction seen in experiment, see Fig.2.

\section{Comparison with the CDF results}

Let us compare our results with the measurements performed in the CDF experiment at Tevatron [i] D0 and CDF detectors. We chose as gluon content of the pomeron the result of the H1 "fit 1" performed in Ref. [5in] , up to a normalization of the flux determined by comparison with CDF results.

We first compare our results for the dijet mass fraction with the measurement of the CDF collaboration [i $\left[\begin{array}{l}\overline{4} \\ {[}\end{array}\right]$ in double pomeron events. As shown in Fig. 2, the dijet mass fraction spectrum is well reproduced. The CDF measurement could clearly not be described without radiation since the obtained dijet mass fraction would peak near one, up to detector resolution effects.

To be more detailed, a tagged antiproton with $0.035 \leq \xi_{\bar{p}} \leq 0.095$ and $|t|<1 \mathrm{GeV}^{2}$ was required. This quantity is reconstructed using the roman pot detectors installed by the CDF collaboration. After the CDF cuts to tag an antiproton and the fast simulation of the detector, we obtain a cross section of $14.4 \mathrm{nb}$, to be compared with the CDF measurement of $43.6 n b$. We thus scale up our cross-sections by a factor $43.6 / 14.4 \sim 3$. 


\section{Predictions for Higgs boson production}

We can now give predictions for the Higgs boson production cross sections in double diffractive events, by scaling our results by the above determined factor . The results are given in Table 1, first column. We note the high values of the cross-sections, which predict ${ }^{1}$ more than 10 events per $\mathrm{fb}^{-1}$ for a Higgs boson mass below $140 \mathrm{GeV}$.

After interfacing the generator with SHW [i] observed in the experiments. The experimental resolution and acceptances of the roman pot detectors have been chosen to be similar to the $\mathrm{D} 0$ ones for dipole detectors, namely the $t$-resolution is $0.1 \sqrt{t}, t$-acceptance $|t| \leq 0.5 \mathrm{GeV}^{2}, \xi$-resolution $0.2 \%$, and the $\xi$-acceptance $100 \%$ if $\xi>0.04,0 \%$ if $\xi<0.01$ and linear between 0 and $100 \%$ if $0.01<\xi<0.04$ [i. tagging efficiency (see column 2 of Table 1) is quite good if one uses dipole detector on each side. To be able to trigger these events, some activity inside the central detector will be required, and we give in Table 1, third column, the number of events after requiring at least two jets of $p_{T}>30 \mathrm{GeV}^{2}$.

To enhance the signal to background ratio, it is possible to cut on the proton and antiproton tagged energy at $930 \mathrm{GeV}$, on the jet topologies (the jets coming from Higgs boson events are more central) and on the reconstructed mass distribution using the missing

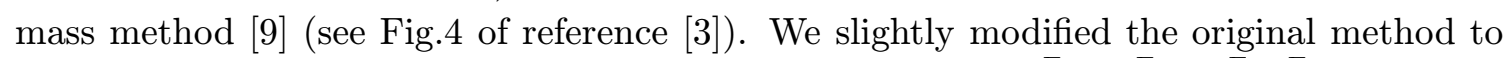
partly take into account radiation and define $M_{H}=\sqrt{\xi_{p} \xi_{\bar{p}} s} \frac{E_{j e t 1}+E_{j e t 2}+E_{p}+E_{\bar{p}}}{2 E_{\text {beam }}}$ where the $E_{\text {jeti }}$ are the leading two jets energies, $E_{p}$ and $E_{\bar{p}}$ the tagged $p$ and $\bar{p}$ energies. We notice that the missing mass method is not working so nicely when radiation is included. It is however still a competitive method to reduce background and reconstruct the Higgs boson mass $^{3}$. Since we obtain quite high cross-sections, other Higgs boson decay channels with smaller branching ratios, like $H \rightarrow \tau^{+} \tau^{-}$(about 10\% of Higgs boson decay, see table 1) or $H \rightarrow W^{+} W^{-}$are of very high interest since the expected background is very small. The background over signal ratio will be studied in more detail in Ref [3]

\section{Acknowledgments}

These results have been obtained from a fruitful collaboration with M.Boonekamp and R.Peschanski.

\footnotetext{
${ }^{1}$ The expected luminosity is between 20 and $25 \mathrm{fb}^{-1}$ per experiment for run II.

${ }^{2}$ This allows us to enhance our signal/background ratio (the $b \bar{b}$ diffractive background without any cuts is estimated to be about $2.310^{7}$ events per $\mathrm{fb}^{-1}$, and becomes about $8.310^{4}$ events after those $p_{T}$ cuts), as shown in Fig. 3 c,d. When we compare Fig. 2 c,d, we also note that radiation is more important for dijet events than for Higgs boson events, since the distribution for dijet events is more shifted to the left after radiation than the Higgs boson one. Hence, the $p_{T}$ cut is more efficient after radiation.

${ }^{3}$ Due to radiation effects which escape mostly into the beam pipe, the missing mass method cannot work as it stands and is modified by radiation. However, it will be very useful to perform constrained fits and to have different ways of reconstructing the Higgs boson mass.
} 


\begin{tabular}{|c||c|c|c|c|c|}
\hline$M_{\text {Higgsboson }}$ & $(1)$ & $(2)$ & $(3)$ & $(4)$ & $(5)$ \\
\hline \hline 100 & 26.6 & 18.5 & 5.7 & 1.9 & 0.2 \\
110 & 21.6 & 14.0 & 5.3 & 1.3 & 0.7 \\
120 & 17.4 & 9.8 & 4.8 & 1.0 & 1.9 \\
130 & 13.8 & 6.1 & 3.2 & 0.6 & 3.3 \\
140 & 10.6 & 2.9 & 1.8 & 0.3 & 4.2 \\
150 & 8.0 & 1.0 & 0.8 & 0.1 & 5.0 \\
160 & 5.7 & 0.2 & 0.1 & 0.0 & 4.5 \\
170 & 3.7 & 0.0 & 0.0 & 0.0 & 2.9 \\
\hline
\end{tabular}

Table 1: Number of Higgs boson events for $1 \mathrm{fb}^{-1}$. The first column gives the number of events at the generator level (all decay channels included), and the other columns after a fast simulation of the detector. The second colum gives the number of events decaying into $b \bar{b}$ tagged in the dipole roman pot detectors (see text), the third one requiring additionally at least two jets of $p_{T}>30 \mathrm{GeV}$, the fourth one gives the number of reconstructed and tagged events when the Higgs boson decays into $\tau$, and the fifth one when the Higgs boson decays into $W^{+} W^{-}$(in this channel, the background is found to be negligible).

\section{References}

[1] J.D. Bjorken, Phys.Rev. D47 (1993), A. Bialas and P.V. Landshoff, Phys. lett. B256 (1990) 540, A. Bialas and W. Szeremeta, Phys. lett. B296 (1992) 191, A. Bialas and R. Janik, Zeit. für. Phys. C62 (1994) 487.

[2] Higgs boson: J-R Cudell and O.F. Hernandez, Nucl. Phys. B471 (1996) 471; E.M. Levin hep-ph/9912403 and referencies therein; V.A. Khoze, A.D. Martin and M.G. Ryskin, Eur. Phys. J. C19 (2001) 477 and references therein.

Dijets: J. Pumplin, , Phys. Rev. D52 (1995) 1477; A. Berera and J.C. Collins, Nucl. Phys. B474 (1996) 183; A.D. Martin, M.G. Ryskin and V.A. Khoze, Phys. Rev. D56 (1997) 5967; A. Berera, Phys. Rev. D62 (2000) 015015;

Higgs boson and dijets: V.A. Khoze, A.D. Martin and M.G. Ryskin, hep-ph/0006005; V.A. Khoze, hep-ph/0105224 and references therein.

[3] M. Boonekamp, R. Peschanski and C. Royon, accepted by Phys. Rev. Lett.

[4] T. Affolder et al., CDF Coll., Phys. Rev. Lett. 85 (2000) 4215.

[5] C. Royon, L. Schoeffel, J.Bartels, H.Jung, R.Peschanski, Phys. Rev D63 (2001) 074004.

[6] B.L. Combridge and C.J. Maxwell, Nucl. Phys. B239 (1984) 429.

[7] SHW, a fast simulation package for DO and CDF detectors, see www.physics.rutgers.edu/jconway/soft/shw/shw.html.

[8] Proposal for a Forward Proton Detector at D0, D0 Collab. (1997), Proposal P-900 to Fermilab PAC.

[9] M.G. Albrow and A. Rostovtsev, hep-ph/0009336. 


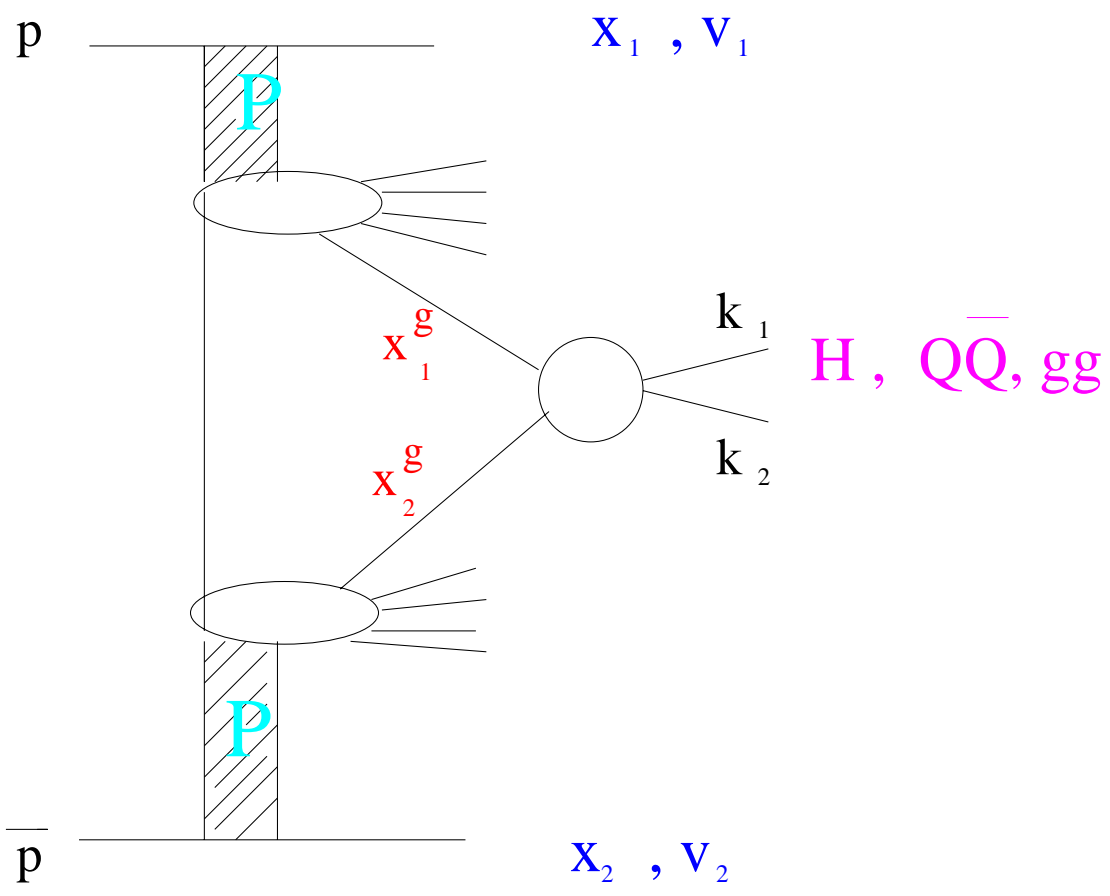

Figure 1: Inclusive production schemme. $x_{i} \equiv 1-\xi_{i}, v_{i}$ are the longitudinal and transverse 2-momenta of the diffracted (anti)proton, $x_{i}^{g}$, the Pomeron fraction momentum brought by the gluons participating in the hard cross-section, $k_{i}$, the transverse 2-momenta of the outgoing jets in the central region from quarks, gluons or the $\bar{b} b$ decay products of the Higgs boson. 


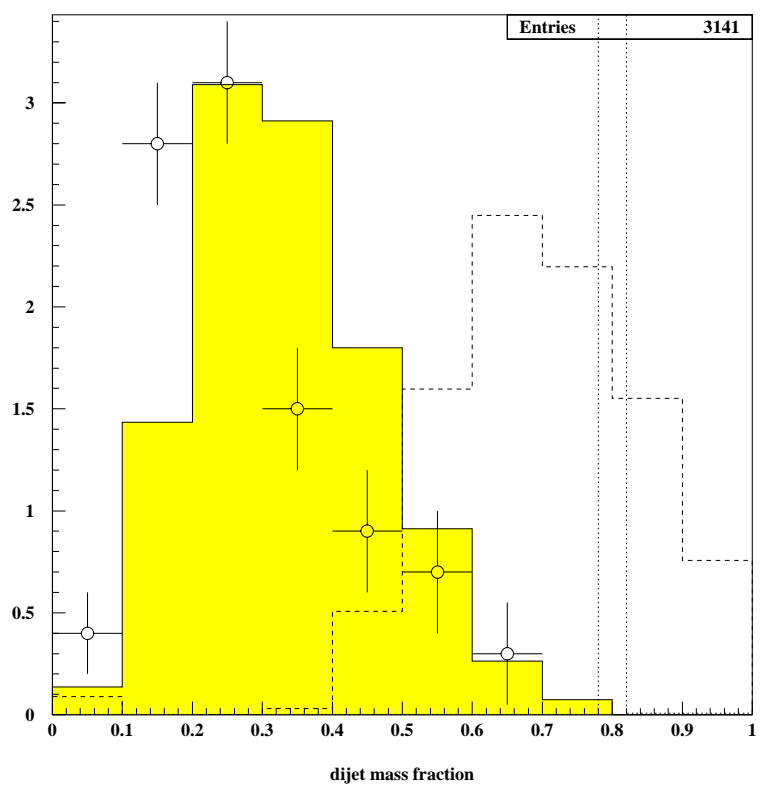

Figure 2: Comparison of the dijet mass fraction obtained in our model and CDF data (open circles). With radiation: The shaded distribution is the dijet distribution after radiation and simulation of the detector.

Without radiation: Dotted line: distribution at generator level; Dashed line: after simulation of the detector. 